\title{
Ion Beam Assisted Deposition
}

\author{
B.R. Nielsen \\ Danfysik A/S, Jyllinge, Denmark
}

Ion implantation into semiconductors for electronic applications represents by far the most widespread industrial use of ion beam technology. In this application, the ability to control the energy, direction, intensity, and beam purity is of extreme importance. To a smaller but still increasing extent ion implantation is used in industry for improving the surface properties such as hardness, wear resistance, friction, fatigue life, and corrosion resistance of metals, ceramics and polymers. The main advantages of ion implantation as a surface treatment are that it introduces no dimensional changes of the substrate, it does not require a high substrate temperatures, the process parameters are well controlled, the implanted species are alloyed within the substrate, and it is possible to obtain surface alloy compositions beyond traditional solubility limits.

The coating of material surfaces with thin films by means of physical vapour deposition (PVD) is another well established industrial process used to harden surfaces or to protect them from corrosion. It has the advantage of enabling the production of several micron thick films of outstanding characteristics such as, for example, the well-known gold-coloured and very hard TiN coating on machining tools. By comparison, layers modified by ion implantation are only a fraction of a micron thick. Drawbacks of the PVD coating technique are that the substrate must be kept at an elevated temperature to make high-quality coatings, and that adhesion of the film to the substrate can be poor. Failure of adhesion can be caused by a difference between thermal expansion coefficients of film and substrate, residual stress in the film, or the presence of impurities or oxides on the substrate surface.

With ion-beam assisted deposition (IBAD) both processes - ion implantation and physical vapour deposition - assist each other in a way that not only eliminates the drawbacks of each of the individual processes, but also creates novel features that neither can produce separately.

\section{The IBAD Process}

The IBAD process is a combined process in which a thin film formed by deposition on a substrate is bombarded continuously with a directed beam of energetic ions. In this way the ion beam interacts with the outermost few atomic layers of the film as it grows, thereby affecting the growth process or modifying the film properties, such as microstructure, density, composition, hardness, and adhesion to the substrate. Although some intermixing between the

Bjarne Nielsen is the Technical Director and VicePresident for Operations at Danfysik A/S, POB 29, DK-4040 Jyllinge. Educated as a physicist, he joined Danfysik after receiving his Ph.D. in atomic collisions in solids from Aarhus University in 1978.

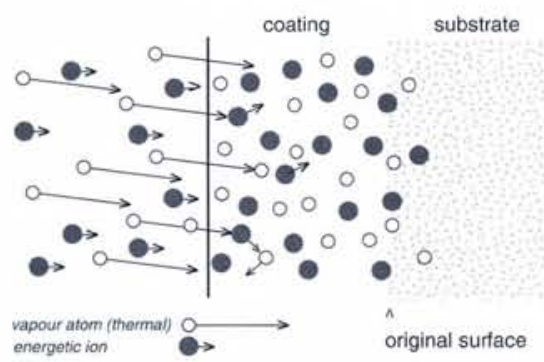

Fig. 1 - Schematic illustration of the principle of ion-beam assisted deposition. Evaporated atoms at thermal energies and energetic ions (100 eV to few keV) arrive at the substrate surface simultaneously. The ion beam only penetrates few atomic layers, but with continuing vapour deposition, the IBAD film may be several microns thick. The ions affect the properties of the film by depositing energy in elastic collisions with the film atoms and/or by reacting with the vapour atoms causing compound formation. Reactive background gas may also be used to influence film properties.

coating and substrate occurs in the initial stages of an IBAD process, it should not be confused with the ion-beam mixing process which involves bombardment of the interface layer between an already deposited film and the substrate with ions of sufficient energy to penetrate the entire film.

The principle of ion-beam assisted deposition is illustrated schematically in Fig. 1. Evaporated atoms and low-energy ions (100 eV to few keV) arrive at the substrate surface simultaneously. Such low-energy particles only penetrate a few atomic layers, but with continued processing the IBAD film
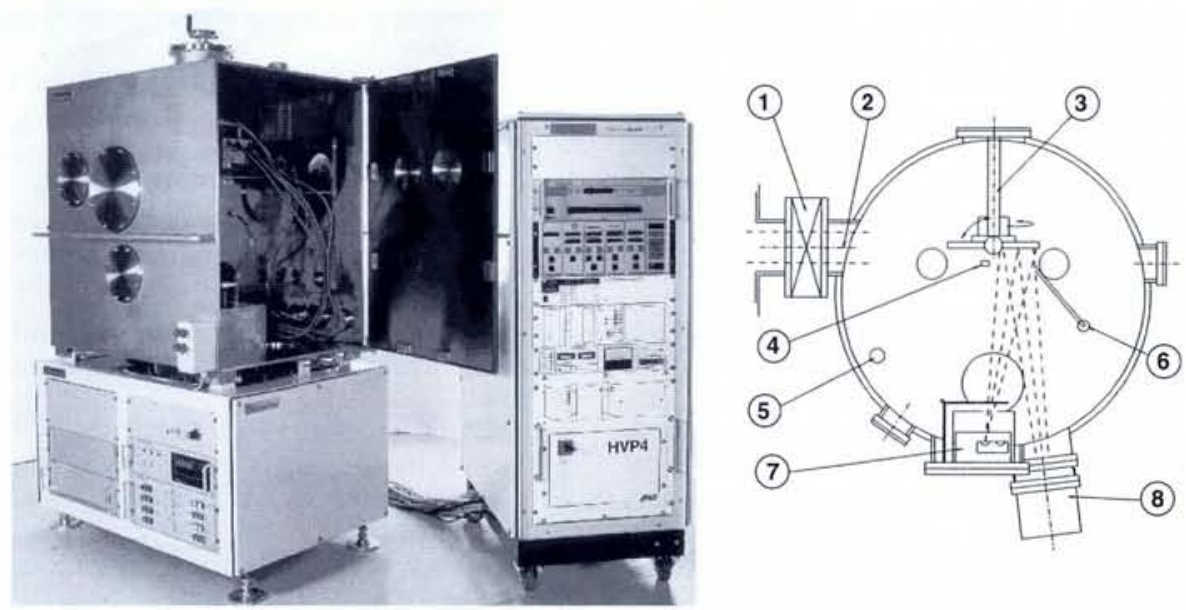

Fig. 2 - A complete set-up for ion-beam assisted deposition, including power supplies, control electronics and vacuum system. The box-shaped IBAD processing chamber has its cover removed. The illustration shows as a vertical section through a cylindrical processing chamber seen from the side. A high-energy ion beam 2 can be can be introduced through the gate valve 1 to impinge on a sample attached to the water-cooled sample holder 3 . The film thickness is measured with a thickness monitor 4 and a gas analyzer 5 monitors the chamber atmosphere. An inlet 6 can be used to introduce a reactive gas and electron-beam evaporated atomic species are produced in a crucible 7 . The low-energy ion beam comes from a broad-beam ion source 8 . may be grown to a thickness of several ties of the film via two mechanisms: a) by depositing energy in the film; and b) by reacting with the vapour deposited atoms cases the latter may include the combined effect of reaction with the atoms of a conrolled inlet or background gas.

A set-up for IBAD processing is illustrated of an electron-beam evaporation system, and a low-energy, broad-beam ion source the angle between the vapour atoms and the ion beam impinging on the sample, a reactive gas into the chamber through a ing is facilitated by means of a quartz crystal deposition rate monitor which is used to sourct the electron beam power; the ion provide a constant preset ion beam current. Finally, a residual quadrupole spectrometer gas analyzer is used to monitor the backshown includes an access port for a beam originating from a high-current ion source. for IBAD pres of higher energy beams duction of non-gaseous (e.g., metallic) ion species into the process. which the reactive gas atoms hit the substrate surface are important parameters for pinging the IBAD process. For the with a current $l$, the ion arrival rate $r_{\mathrm{i}}=I / A e$, where $e$ is the electron charge. An ion beam current density of $160 \mu \mathrm{A} / \mathrm{cm}^{2}$ corresponds vapour atom and background gas arrival rate $r_{\mathrm{a}}$ depend on the partial pressure $p$ and the temperature $T$ as follows: produces the low-energy ion beam. The which is mounted on a rotatable, watercooled sample holder. It is possible to pass

and the atoms 


$$
r_{\mathrm{a}}=p(2 \pi m k T)^{-1 / 2},
$$

where $m$ is the molecular weight and $k$ is Boltzmann's constant. For nitrogen at room temperature, the residual gas pressure corresponding to an arrival rate of $10^{15}$ atoms $/ \mathrm{cm}^{2} \mathrm{~s}$ or $0.5 \times 10^{15}$ molecules/ $\mathrm{cm}^{2} \mathrm{~s}$ is $1.7 \times 10^{-6}$ mbar. A vapour deposition rate of $10^{15}$ atoms $/ \mathrm{cm}^{2} \mathrm{~s}$ corresponds to a typical film growth rate of $2 \mathrm{~A} / \mathrm{s}$.

\section{Film Characteristics and Applications Conventional IBAD}

When thin films are grown by physical vapour deposition at low temperatures the microstructure often shows a columnar structure with columns in the direction of the impinging atoms, causing porosity of the film which is normally undesirable. Raising the deposition temperature above a certain critical value (typically $0.25 T_{m}$, where $T_{m}$ is the film melting temperature) causes this structure to be levelled out owing to surface diffusion. With IBAD, the energy of the ion beam is mainly deposited via elastic ion-atom collisions and the atomic movements caused by the collision cascade inhibit development of the columnar structure. Hence, with IBAD it is possible to create very dense thin films even at low temperatures. This is particularly advantageous when high temperatures modify surface properties.

Another effect of energy deposition by the ion beam is the reduction of residual stress in the film. Residual stress is caused by the film microstructure, different thermal expansion coefficients between film and substrate, and impurities. The stress can be effectively annealed out if the ion/atom arrival rate ratio $R$ is high enough to ensure that each atom of the deposited film is involved in a collision cascade. The critical $R$-value for this to occur depends on the ion energy, but is typically less than 0.01 , i.e., a beam current intensity of less than $1.6 \mu \mathrm{A} / \mathrm{cm}^{2}$ is sufficient for a film growth rate of $2 \AA / s$. Stress annealing represents an important means to avoid cracking or delamination of the film.

Film adhesion can be further improved by using the ion beam to sputter-clean the substrate surface and by intermixing of the film and substrate atoms (the latter requires high beam energies to ensure sufficiently deep penetration into the substrate). Using these approaches, $\mathrm{MoS}_{2}$ films with extremely good adhesion can be produced for dry lubrication purposes.

\section{Reactive IBAD}

In the examples described above the primary role of the ion beam is to transfer momentum and energy to the film and substrate atoms. In such cases, the process normally uses noble gas ions, and typical ion/atom arrival rate ratios are much less than one. Low- and medium-current ion beams are therefore sufficient. In the cases where the ion beam supplies atoms for compound generation (so called reactive IBAD) the ion species is obviously of importance. Furthermore, the $R$-value is a much more critical parameter for obtaining the correct stoichiometry and the desired film properties. High ion currents are required for this application since ion and atom arrival rates must be similar in magnitude. Hence, a versatile ion-beam system capable of produc-

\section{DANFYSIK}

\section{From Source to Target}

Danfysik is considered unique as it serves customers from around the world with a broad range of physics-based equipment that is built in Denmark. Operating as a private company, it was founded in 1964 by E. Jespersen, the Managing Director.

Danfysik has built its reputation on taking particle beams as the common denominator to supply well-established products (ion sources, beam diagnostic equipment, electromagnets, electrostatic devices, magnetic power supplies, implanters), building blocks for numerous beam generation and beam transport systems, and tailor-made systems and components (spectrometers, storage-ring magnets, electron coolers, insertion devices, microbeam equipment, ultrastable and fast-ramping power supplies).

Specialised in working in close collaboration with customers, the company employs a team of physicists, engineers and designers who also offer a complete range of consultancy and design services. Manufacturing operations were moved in 1990 to a new, purpose-built factory in Jyllinge which also houses the company's headquarters. There are currently some 60 employees, including 17 physicists and engineers. The philosophy is to have outside contractors manufacture as much as possible to ensure sufficient capacity to handle major orders. Most accelerator laboratories around the world have purchased equipment from Danfysik.

After offering ion-beam implanting systems to the semiconductor industry for several years, Danfysik started in 1989 to manufacture systems for surface engineering. It is one of the few companies which manufacture high-current systems. Danfysik has participated in joint research projects in the field of surface modification under European programmes such the European Union's Brite/Euram. The following institutes have been supplied with ion implanters for surface engineering:

Danish Technological Institute (Aarhus, Denmark; 1987)

LNETI (Lisbon, Portugal; 1992)

IWT (Bremen, Germany; 1992)

CNRSM (Brindisi, Italy; 1992)

Joint Research Centre (Ispra, Italy; 1989)

INSAMET (San Sebastian, Spain; 1993) FZR (Rossendorf, Germany; 1994)

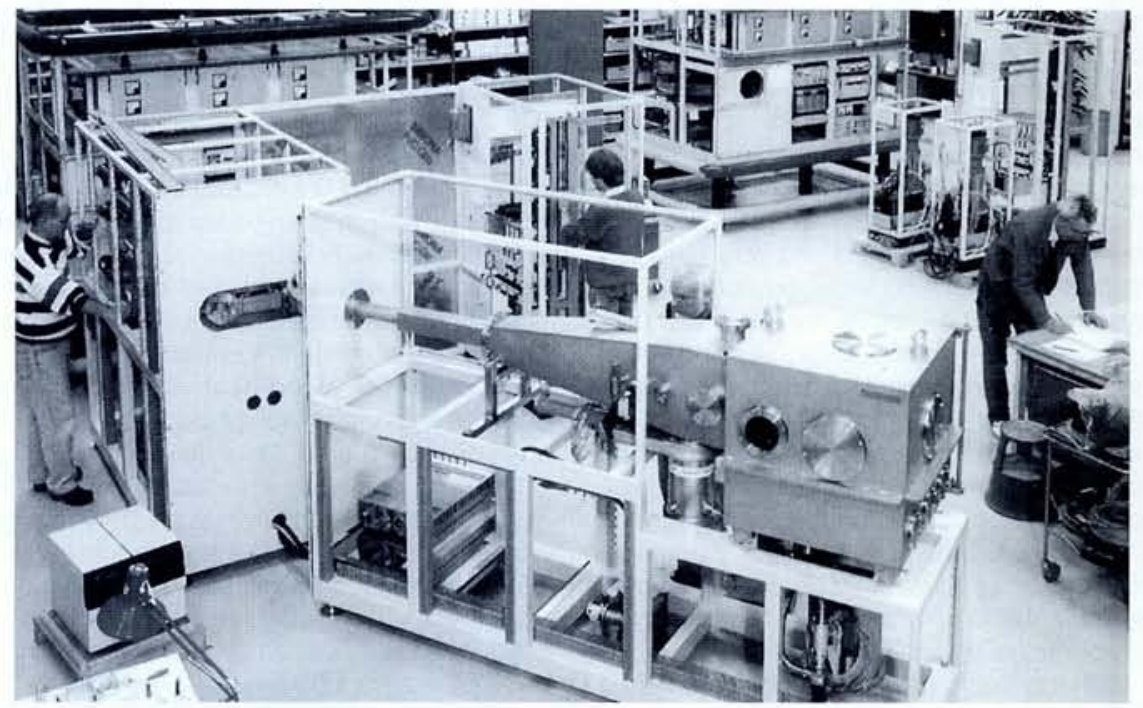

A high-current ion implanter with one beam line under construction in the Danfysik factory at Jyllinge. An ion source with extraction followed by mass analysis generates ion beams that are accelerated in the accelerating tube coupled to the processing chamber shown on the right. A magnetic quadrupole and a beam scanning system placed before the chamber are used manipulate the beam.

ing high beam currents of many different gaseous (and if possible metallic) ions is required.

Using the reactive IBAD technique it is possible to make dense films of TiN or AIN with good adhesion by evaporating $\mathrm{Ti}$ or $\mathrm{Al}$ while bombarding the film with a nitrogen beam having an intensity corresponding to $R=1$. They may also be produced by bombarding the metal film with a noble gas beam, e.g., $\mathrm{Ar}^{+}$, while applying the nitrogen as background gas. Evaporation of $\mathrm{Zr}$ under oxygen beam bombardment can produce very dense oxide coatings for optical applications. Other IBAD techniques still in the research stage include the production of extremely hard and chemically inert diamond-like coatings of carbon and cubic boron nitride.

\section{Perspectives}

The first industrial-scale application of the IBAD technology has been in the production of optical antireflective coatings, where the improved adhesion and density relative to non-ion based deposited coatings is a great advantage. Other applications, such as corrosion protection, wear resistance and diamond-like carbon coatings are also finding industrial applications.

With the large number of possibilities for engineering new, low-temperature coatings in a controlled way, the IBAD technique will undoubtedly lead to further research and development work. As a consequence the technique will find new applications, where the specific advantages of IBAD films are in demand. 